\title{
Reconstruction of the trachea with a tubed radial forearm free flap
}

\author{
B. R. Beldholm, MBBS, BSc(Med) ${ }^{\text {a }}$ \\ M. K. Wilson, MBBS, BSc(Med), FRACS ${ }^{a}$ \\ R. M. Gallagher, MBBS, FRACS ${ }^{b}$ \\ D. Caminer, MBBS, FRACS ${ }^{c}$ \\ M. J. King, MBBS, FFARCS ${ }^{d}$ \\ A. Glanville, MD, FRACPe
}

From Cardiothoracic Surgery, ${ }^{\mathrm{a}}$ Department of Otolaryngology-Head and Neck Surgery, ${ }^{\mathrm{b}}$ Department of Plastic and Reconstructive Surgery, ${ }^{\mathrm{c}}$ Anaesthetics, ${ }^{\mathrm{d}}$ and Thoracic Medicine, ${ }^{e}$ St Vincent's Hospital, Sydney, Australia.

Received for publication Jan 4, 2003; accepted for publication Jan 22, 2002.

Address for reprints: Dr Bernard Richard Beldholm, St Vincent's Hospital, PO Box 689, Miranda NSW 1490, Australia (Email: Beldholm@yahoo.com).

J Thorac Cardiovasc Surg 2003;126:545-50

Copyright (C) 2003 by The American Association for Thoracic Surgery

$0022-5223 / 2003 \$ 30.00+0$

doi:10.1016/S0022-5223(03)00357-X
Background: We present the case of a patient with adenoid cystic carcinoma of the trachea who had $60 \mathrm{~mm}$ of the trachea excised and reconstructed with a stented radial forearm free flap. The patient was well in the immediate postoperative period with good function of the neotrachea. Problems developing after the reconstruction included proximal stricture, sputum retention, and recurrent pneumonia.

Result: The patient died of malignant hypercalcemia 16 months after the reconstruction. To our knowledge this is the first reported case of a total tracheal resection and reconstruction with a combination of free tissue transfer and internal stenting.

Conclusion: We conclude that tracheal reconstruction has the potential to provide a reliable airway in patients not able to be reconstructed with a primary anastomosis.

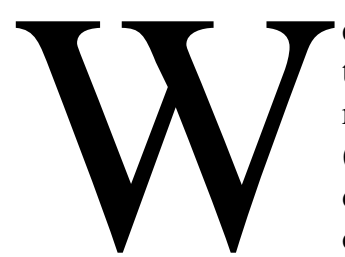

e report a case of adenoid cystic carcinoma of the trachea that was resected and the trachea reconstructed with a tubed radial forearm free flap (RFFF) supported by Ultraflex stents (Boston Scientific, Boston, Mass). According to Azar, Abdul-Karim, and Tucker, ${ }^{1}$ the first reported case of adenoid cystic carcinoma was in 1762 by Morgagni. Billroth, as cited by Pearson and associates, ${ }^{2}$ first described the characteristics and pathology of adenoid cystic carcinoma in 1859. Ever since then, tracheal tumors have provided a diagnostic and treatment challenge. Tracheal tumors are uncommon, with an estimated incidence of 2.7 new cases per million per year. ${ }^{3}$ The majority of these patients have progressive dyspnea, wheeze, and/or persistent cough, which is often initially misdiagnosed as asthma or bronchitis. It is not until late in the course of the illness that other symptoms such as stridor, hemoptysis, dysphagia, or hoarseness raise the possibility of an obstructive lesion. ${ }^{1,4}$ Tracheal tumors present a diagnostic challenge because of their rarity and nonspecific symptoms. When the tracheal tumor is eventually discovered, it has usually nearly occluded the trachea and the possibility of cure is severely reduced. Tracheal tumors can be divided into 3 major groups; adenoid cystic carcinoma, squamous cell carcinoma, and miscellaneous. Adenoid cystic carcinoma accounts for 30 to $40 \%$ of tracheal tumors and has some distinct features.

Adenoid cystic cancer is a locally invasive tumor that has a long progressive course. It is the second most common malignant salivary gland tumor. Local recurrence is usually the cause of death. Pulmonary metastases are most common, but metastases to brain, bone, liver, skin, kidney, abdomen, and heart have been described. ${ }^{1}$ Macroscopically, it appears as a circumferential nodule with a smooth surface. The macroscopic appearance is quite deceptive, and it usually extends far beyond the macroscopic limit. The only treatment that is effective is surgical resection with radiotherapy having only an adjuvant role. Even if the cancer is 


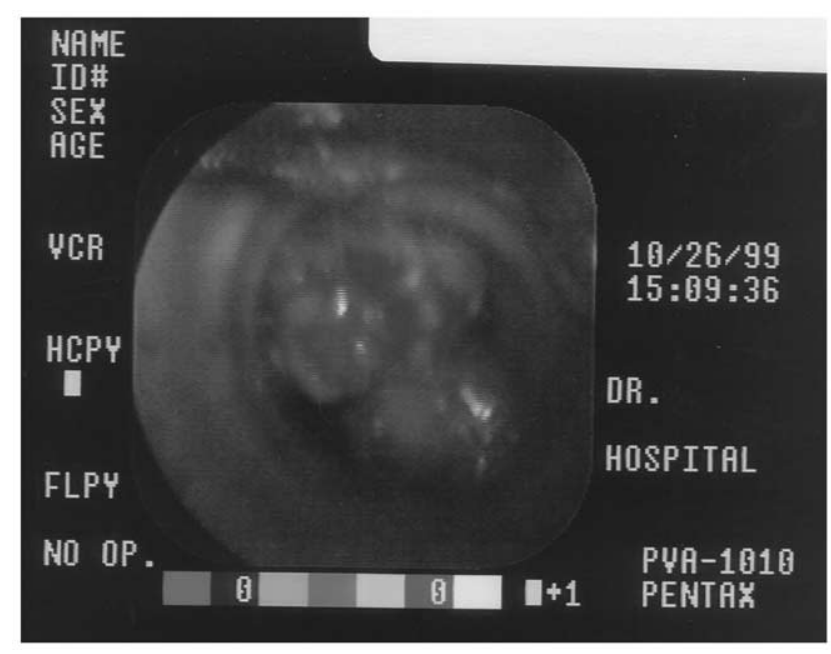

Figure 1. Tracheal tumor before debulking and definitive operation.

incompletely excised, prolonged palliation can be achieved. ${ }^{1}$ Local recurrences can occur many years later, and recurrences after 10 to 15 years have been reported. Therefore, long-term follow-up is required.

Once the tracheal tumor has been diagnosed, a decision has to be made as to what treatment can be offered. A number of case series with resection and primary anastomosis have demonstrated a good success rate, with good palliation or cure. The only real possibility of cure in a tracheal cancer is surgery, because radiotherapy is not curative. Extensive circumferential defects up to $50 \%$ and in rare instances larger defects can be closed primarily. Mobilization and suprahyoid release of the larynx may be required. Reconstruction of extensive circumferential tracheal defects with autologous tissue has until now proved beyond surgical possibility. For the patient requiring total surgical resection of the trachea for tumor clearance, tracheal prostheses or mediastinal tracheostomy represent less than satisfactory methods for maintaining airway continuity. We present the first reported case to our knowledge of total tracheal resection and reconstruction using a combination of autologous free tissue transfer and internal stenting.

\section{Case Study}

A 43-year-old woman had increasing respiratory distress over 9 weeks. She had difficulty with inspiration. Symptoms were worse at night, when lying down, and at rest. On examination she had marked inspiratory and expiratory stridor, with widespread expiratory wheezes on auscultation. Examination did not show lymphadenopathy or hepatosplenomegaly. Lack of response to initial treatment for asthma led to the performance of chest computed tomography (CT), which revealed near complete obstruction of the trachea by a polypoid mass.
Examination with a rigid bronchoscope showed a 1- to 2-cm mass $3 \mathrm{~cm}$ from the carina (Figure 1). Laser debulking was undertaken and a Dumon (Novatech, Aubagne, France) stent inserted. Histologic study showed an adenoid cystic carcinoma with high-grade areas. The patient had marked relief of her symptoms from this initial procedure.

Before the planned definitive operation, the patient underwent mediastinal endoscopic ultrasound, positron emission tomography (PET), and a chest CT scan. Endoscopic ultrasound showed a normal mediastinum, no nodal involvement, and no sonographic features of esophageal wall infiltration. CT showed the tracheal tumor involving 3 to 4 $\mathrm{cm}$ of the midtrachea. PET scanning demonstrated no metastatic disease. Bronchoscopy before the operation confirmed that there was no macroscopic evidence of tumor involving the upper and lower margins of the trachea.

\section{Operation}

The operation was carried out in 4 phases:

1. Upper tracheal mobilization and RFFF harvest, supine with double lung ventilation.

2. Right thoracotomy, tracheal resection, neotrachea placement, and intubation, decubitus with left single lung ventilation.

3. Neotrachea microvascular anastomosis, neck closure, and split skin graft from thigh, supine with left single lung ventilation.

4. Thoracotomy closure and skin graft to arm, decubitus double lung ventilation.

The Dumon stent (Bryon Corp, Woburn, Mass) was too small to admit a double-lumen endotracheal tube, so a single-lumen size 6 armored endotracheal tube was passed into the conventional position for double lung ventilation and then passed into the left main bronchus for left single lung ventilation.

Phase 1. A utility neck flap was raised and the strap muscles separated. The trachea was then exposed in the neck and mobilized with preservation of the recurrent laryngeal nerve. Simultaneously, the left RFFF was mobilized and a flap of skin 13 by $6.5 \mathrm{~cm}$ was elevated. The flap was raised by standard techniques and the radial artery divided at the distal end of the wrist. The cephalic vein and deep communicating veins were harvested to the cubital fossa. A 5-mm border around the flap was deepithelialized for tubing at the distal and proximal anastomosis. The flap was then tubed around the Ultraflex stent (Boston Scientific Co, Boston, Mass) with continuous 4-0 Monocryl suture (Ethicon, Inc, Somerville, NJ).

Phase 2. The patient was rolled left side down and the chest entered through a posterolateral thoracotomy in the right fourth intercostal space. The single-lumen tube was advanced from the trachea into the left main bronchus for single lung ventilation. The intrathoracic trachea was then 
mobilized by dividing the azygos vein and the vagus nerve. The distal trachea was divided and the left main bronchus directly cannulated via the thoracotomy while the endotracheal tube was withdrawn into the larynx. Single lung ventilation was continued in this fashion while the remainder of the trachea was then mobilized from above and below. The trachea was excised 2 rings below the cricoid and 1 ring above the carina. Frozen section of the proximal ring was clear of tumor but the distal ring was not, so the remaining distal ring was excised and was disease free on frozen section.

The neotrachea was transferred into the thorax and the pedicle divided and delivered into the neck, ensuring no torsion. The proximal and distal anastomoses were commenced, and the posterior wall or membranous trachea was sutured to the neotrachea with 3-0 Prolene continuous sutures (Ethicon). The Ultraflex stent was flush with and partially incorporated in the distal anastomosis. There was a 3- to 4-mm gap between the proximal anastomosis and the Ultraflex stent, which had to be covered by a second Ultraflex stent deployed from below to cover the gap and allow the endotracheal tube to be advanced via the neotrachea into the left main bronchus. The anterior wall of the distal and proximal tracheal anastomoses was then completed. The thoracotomy wound was covered with an OpSite dressing (Smith \& Nephew, London, United Kingdom) and the patient rolled back to the supine position.

Phase 3. Microvascular anastomoses were performed with 8-0 monofilament nylon interrupted sutures and microvascular staples (medium; Auto Suture Company Division, United States Surgical Corporation, Norwalk, Conn.). The radial artery was joined to the external carotid artery as an end-to-side anastomosis and the deep and superficial veins of the flap to the internal jugular vein as an end-to-side anastomosis. The strap muscles closed anteriorly in front of the anastomosis. A single Jackson Pratt drain was placed in the neck and the remainder of the neck wound closed in layers. A split skin graft was then harvested and the radial forearm donor site was split skin grafted and supported with a back slab.

Phase 4. The patient was then rolled left side down again and the thoracotomy wound explored. The singlelumen tube was withdrawn back up into the distal trachea; the anastomosis was airtight and tested for leaks with warm water. The pedicle was pulsing nicely and the tracheal conduit appeared well vascularized. Two chest drains were placed and the thoracotomy wound closed with pericostal and subcuticular sutures. The patient was rolled supine and the single-lumen tube exchanged for a larger tube allowing bronchoscopy and tracheobronchial direct suction (Figure 2).

\section{Comment on the Operation}

The 2 Ultraflex stents used were uniquely suitable for this reconstruction and probably no other commercially avail-

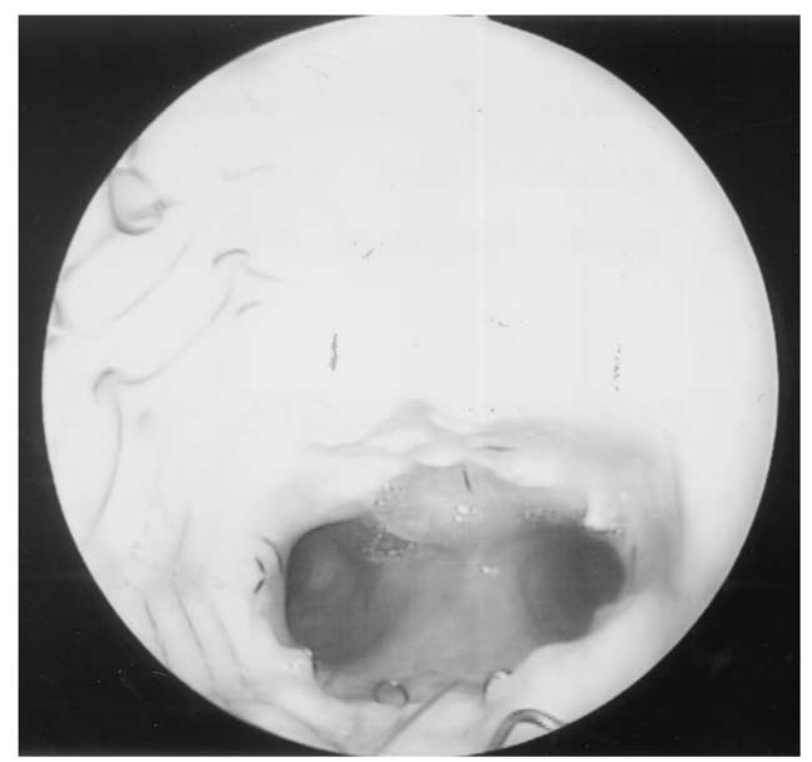

Figure 2. Distal view of anastomosis with the Ultraflex stent in situ and showing the suture line and the carina.

able stent would have functioned as well. The 2 stents were used as a backbone for the reconstruction and were sutured together to provide optimal function. The use and properties of this stent are further discussed below.

Histologic examination showed an adenoid cystic carcinoma $40 \mathrm{~mm}$ in length and a maximum thickness of $10 \mathrm{~mm}$. On the initial specimen the tumor was $9 \mathrm{~mm}$ from the proximal margin with the distal margin involved; after further excision the distal margin was cleared. In total there were 12 lymph nodes in the specimens sent to pathology and they were all confirmed to be clear of tumor.

\section{Postoperative Course}

The patient was extubated at the end of the procedure after the placement of an epidural catheter afforded good analgesia and respiratory function. The patient was mobilized the following day and the chest drains removed on postoperative days 2 and 3. Respiratory function was maintained with aggressive physiotherapy and analgesia despite some early problems with atelectasis and sputum retention.

The initial bronchoscopic examination showed good healing of the anastomoses and remarkably good function of the neotrachea, especially during coughing when partial tracheal closure mimicked the physiology of the native trachea. The patient was well and discharged with only a minor nonproductive cough.

The patient returned for bronchoscopic examination 1 month after the operation. At this stage a cough productive of red-brown sputum had developed. Sputum sample showed Staphylococcus aureus. Bronchoscopy demonstrated multiple areas of granulation tissue growing into the 


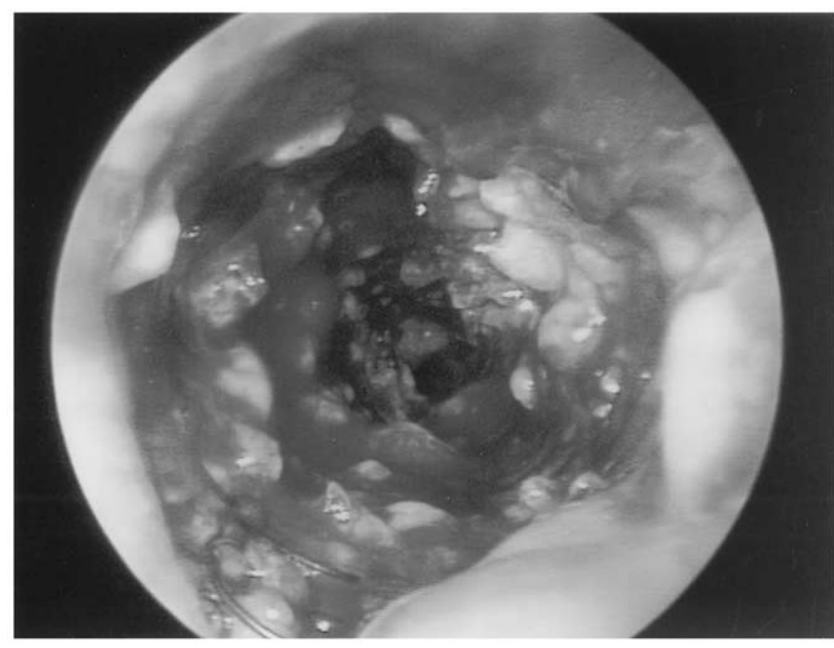

Figure 3. Exuberant granulation tissue growing in through the stent postoperatively.

trachea from the flap covering the stent (Figure 3). Granulation tissue was cauterized and resected at this session. The patient was discharged in good health the next day.

Postoperative radiotherapy was commenced 40 days after the reconstruction. A total of $52 \mathrm{~Gy}$ was delivered in 26 fractions to the mediastinum and lower parat of the neck. She had mild esophagitis, right neck pain associated with swallowing, and a mild erythematous skin reaction of the lower neck, all of which resolved.

During the year after the operation, the patient was well but continued to have a persistent dry cough. A subglottic stricture (Figure 4) also developed just above the stent, which had to be dilated on multiple occasions. Bronchoscopic examinations were performed every 5 to 6 weeks. There were multiple admissions for $S$ aureus pneumonia and she had problems with sputum plugging culminating in 1 episode of respiratory arrest necessitating intubation.

Severe pain in her left shoulder also developed, necessitating high-dose narcotics. PET scan showed an infiltrative process in both lungs thought likely to represent an inflammatory disease rather than malignancy. Bone scan demonstrated uptake in the right ischium, superior sacrum, thoracic lumbar spine, T3, T10 vertebrae, and fifth, sixth, and tenth ribs. A left pleural effusion developed, but cytologic examination did not demonstrate malignant cells. Sixteen months after the initial operation, she was admitted to the hospital with increasing bone pain predominantly in the midthoracic and shoulder regions, increasing dyspnea, and increasing malaise. She had marked weight loss, hypercalcemia, and anemia. Chest radiography showed a diffuse infiltrate throughout the left lung. In consultation with the family and patient, the decision was made that further invasive ventilatory support was not appropriate. Her respi-

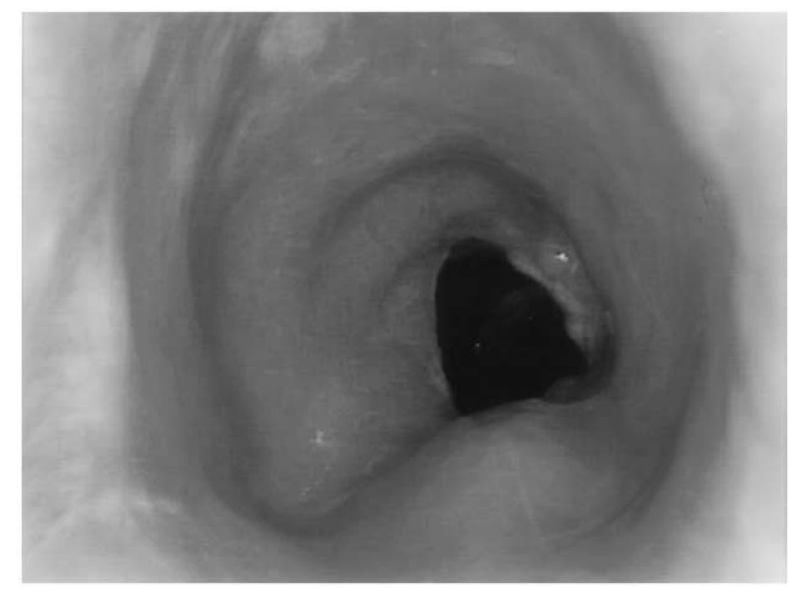

Figure 4. Postreconstruction view of the trachea showing the proximal stenosis.

ratory status progressively deteriorated and she died shortly thereafter. An autopsy was not performed.

\section{Discussion}

Tracheal tumors are uncommon and there has therefore been a slow progression in the treatment and management of these tumors. The best option for managing these tumors is surgical resection. Before the 1960s it was believed that only $2 \mathrm{~cm}$ of the trachea could be resected and primary anastomosis achieved, but with new techniques it is today possible to resect in some instances more than $50 \%$ of the trachea and still obtain primary anastomosis. ${ }^{4-6}$ Beyond these limits it is necessary to replace the trachea with a prosthesis. This remains a therapeutic challenge.

The trachea is a perfectly constructed organ ideally suited for its functional demands. It is a rigid airtight structure that can vary its diameter for optimal conduction of air, it has the ability to clear secretions, and it senses any foreign material. As with any organ, prostheses or reconstructions can only crudely match what nature has constructed. An ideal tracheal prosthesis should have lateral rigidity, vertical flexibility, an airtight lumen, reliable healing, and a continuous lining with respiratory epithelium. ${ }^{7}$ So far, no method or material has been described for fulfilling all these requirements.

Recently Grillo ${ }^{8}$ did an extensive review of tracheal replacement with artificial and autologous materials; this review concludes that there has been a very poor success rate so far. Constructing a functional tracheal conduit that provides good function with minimal complications has proven difficult, as can be attested by the wide range of materials used in the attempts. So far a high mortality and morbidity rate has been apparent in all tracheal prosthetic reconstructions, although some of the individual experimental studies hold promise. 
Initially foreign materials were used, such as stainless steel, steel coils, silicone, Teflon, and Marlex mesh. These replacements had a number of serious problems. They were not incorporated into the local tissues; they migrated, dislodged, got infected, and became obstructed. A particular problem was the formation of granulation tissue at the anastomosis, which would lead to stenosis and obstruction. The trachea is not a sterile structure and so infections tended to develop around the anastomosis. These materials where also very stiff, which meant that they would erode into the surrounding structures and a high mortality rate from brachiocephalic artery ruptures were noted early on. Early reports of replacement with Marlex mesh would have a mortality rate of up to $70 \%$ in the early postoperative period. ${ }^{2,5,9}$ So far the majority of trials with foreign materials have had poor results. ${ }^{8}$

In an attempt to overcome the problems of nonepithelialization, granulation tissue formation, and migration of the conduit, trials have been conducted with porous materials as well as cadaveric nonviable tissues. Trials both in human subjects and in animals have shown that excessive granulation tissue leads to stenosis, and the need for frequent bronchoscopic treatments has been a continuing problem. ${ }^{8,10}$ Some apparent experimental success has been achieved with the porous type of tracheal prosthesis, ${ }^{10,11}$ where variable lengths of conduits were incorporated into animals with reepithelialization occurring in the majority of cases. These materials have yet to have any impact in human studies.

It is recognized that autologous tissue provides better blood supply and primary healing, especially in areas with previous surgery or irradiation. The difficulty with reconstructing a circumferential tracheal defect has been to design a flap with intrinsic or added rigidity to prevent inward collapse, stenosis, or obstruction. A number of animal studies have been performed to investigate the feasibility of total tracheal reconstruction using autologous tissue. Jones and colleagues $^{12}$ and Nakayama ${ }^{13}$ have reported the use of a free jejunal microvascular flap to reconstruct both noncircumferential and circumferential tracheal defects in a canine model. The main disadvantages of these flaps are their lack of rigidity and excessive mucous secretion. Nakayama then added an intraluminal silicone $\mathrm{T}$ tube to provide support and showed that cauterization of the mucosa successfully reduced mucous secretion.

A large number of different free grafts have been used for tracheal replacement, ranging from omentum, perichondrial slips, and bladder mucosa to dura mater. ${ }^{8}$ These free grafts have been supported by an equally large number of supports from silicone tubes to prefabricated cartilage rings. In the experience to date none of these has had lasting success. Multistage cutaneous tubes have been used to reconstruct the cervical trachea, but high mortality and mor- bidity rates were noted. Obtaining an airtight seal immediately was also not possible and so reconstruction of the intrathoracic trachea would be difficult. A number of other authors ${ }^{14,15}$ have attempted to prefabricate flaps with cartilage grafts and skin grafts for lining before transfer. In a recent study by Cavadas ${ }^{16}$ using a goat model, a composite cutaneous chondromucosal premolded, prevascularized flap was reconstructed in a 2-staged procedure with subsequent microvascular transfer. In this case, the entire trachea was reconstructed with autologous cartilage and nasal mucosal grafts around a silicone stent implanted into a thigh. The stent was then removed before transfer, creating a total autologous neotrachea. In this study only $30 \%$ of the animals survived. The main disadvantage with prefabrication techniques is that they are multistaged and would be difficult to apply in the clinical setting.

From the above discussion it is apparent that a new approach to tracheal replacements needs to be taken. There has been previous experience in using RFFF to reconstruct in part the trachea and the esophagus. Wheatley and colleagues ${ }^{17}$ used an RFFF to elongate the trachea so as to construct a neotracheostome after resection of recurrent stomal tumors. Four patients were treated by this technique and had a good postoperative course, with none having stomal narrowing or strictures. One patient had venous thrombosis in the graft, which was treated successfully, and subsequently did well. The longest follow-up available in this series is a patient at 38 months after reconstruction who is still doing well. RFFF for pharyngoesophageal reconstructions was described in $1985 .{ }^{18}$ Since then a large number of patients have been treated with this reconstruction with a good success rate. For relevance to tracheal reconstructions, the RFFF has been found to be a highly reliable flap, there is limited donor site morbidity, and a long vascular pedicle is available. ${ }^{18}$

The concept of combining an RFFF with an internal stent in tracheal replacements has not, to our knowledge, been previously attempted. The rationale for using a skin lining is that it would be more robust and able to withstand sheer and abrasion from the stent. This model satisfies almost all of Belsey's criteria for the reconstructed trachea. It provides lateral rigidity, vertical flexibility, and remarkable physiologic compression for clearance of sputum as seen on bronchoscopic examination.

The purpose of the stent in this reconstruction was to provide stability and a patent airway. Other properties that were necessary were lateral flexibility and for the stent not to migrate. It was also important that the stent be able to return to its original shape after being deformed for the reconstruction to work. The Ultraflex stent has all of the necessary properties and was uniquely suitable for use in this reconstruction, as compared with stainless steel stents such as the Gianturco-Z stent and the Wallstent and silicone 
stents such as the Dumon stent. These properties include superelasticity, shape memory, biocompatibility, kink resistance, and fatigue resistance. ${ }^{19}$ It provides a larger diameter for the airway as compared with the Dumon stents, and also if uncovered provides the opportunity for respiratory epithelium to be reformed. ${ }^{19}$ The Ultraflex stent is a selfexpanding stent constructed out of nickel-titanium alloy (nitiniol); when released it contracts in length by $27 \%$ to $40 \% .{ }^{20}$ Nitiniol has the property of superelasticity and shape memory. Superelasticity refers to the ability of nitiniol to be deformed up to $10 \%$ to $12 \%$ and still regain its original size and shape; stainless steel can only tolerate a deformation of up to $0.5 \%$ before changing shape permanently. Shape memory refers to the ability of the device to restore its original configuration in a specific temperature range. ${ }^{21}$ The function of the Ultraflex stent was well demonstrated by the postoperative bronchoscopic examinations, which showed that the stent would be deformed during vigorous coughing but would afterward spring back to its original shape. Problems that have been noted with expandable metal stents have been excessive granulation tissue formation, sputum retention, insufficient stent expansion, and difficulty removing once deployed. ${ }^{22-24}$ The rate of complications with the Ultraflex stent as used in the esophagus and the tracheobronchial tree has been noted to be substantially lower than with other types of stents. ${ }^{19,23}$

The use of the microvascular flap provides an airtight lumen and good vascularized tissue for reliable healing. The only criterion not satisfied is the requirement for a continuous lining with respiratory epithelium for the neotrachea. A suggested modification to the procedure would be to deepithelialize the RFFF and seed the dermis with respiratory mucosal epithelial cells. The theoretical benefits of such a lining relate to clearance of secretions using ciliary action. The absence of mucosal lining may lead to pooling of secretions and subsequent infection. Initially our patient was shown on both physical and bronchoscopic examination to have good clearance of secretions and a vigorous cough reflex. The patient's subsequent course was complicated by recurrent pneumonia and several episodes of sputum plugging, 1 episode necessitating intubation. Obtaining a respiratory mucosal lining may therefore be an important aim in the long-term success of any tracheal replacement.

\section{Conclusion}

Despite a wide range of materials and methods used for replacing the trachea, a reliable method is still to be found. Using the RFFF with an Ultraflex stent has some advantages, such as reliability of closure and good healing, as well as reduced donor site morbidity. There are some problems with the reconstruction such as stricture formation and lack of respiratory epithelium. Overall, the RFFF reconstruction provides a good neotrachea, and future work may prove this to be a viable technique for reconstructing circumferential defects of the trachea that are not able to be closed by primary anastomosis.

\section{References}

1. Azar T, Abdul-Karim FW, Tucker HM. Adenoid cystic carcinoma of the trachea. Laryngoscope. 1998;108:1297-300.

2. Pearson FG, Thompson DW, Weissberg D, et al. Adenoid cystic carcinoma of the trachea. Experience with 16 patients managed by tracheal resection. Ann Thorac Surg. 1974;18:16-29.

3. Mathisen DJ. Primary tracheal tumor management. Surg Oncol Clin N Am. 1999;8:307.

4. Pinsonneault C, Fortier J, Donati F. Tracheal resection and reconstruction. Can J Anaesth. 1999;46:439-55.

5. Pearson FG, Todd TR, Cooper JD. Experience with primary neoplasms of the trachea and carina. J Thorac Cardiovasc Surg. 1984;88:511-8.

6. Regnard JF, Fourquier P, Levasseur P. Results and prognostic factors in resections of primary tracheal tumors: a multicenter retrospective study. The French Society of Cardiovascular Surgery. J Thorac Cardiovasc Surg. 1996;111:808-14.

7. Belsey R. Resection and reconstruction of the intrathoracic trachea. Br J Surg. 1951;38:200.

8. Grillo HC. Tracheal replacement: a critical review. Ann Thorac Surg. 2002;73:1995-2004.

9. Grillo HC. Tracheal tumors: surgical management. Ann Thorac Surg. 1978;26:112-25.

10. Jorge RG, Armengod AC, Caravajal JM. As originally published in 1990: experimental study of a new porous tracheal prosthesis. Updated in 1998. Ann Thorac Surg. 1998;65:1830-1.

11. Teramachi M, Nakamura T, Yamamoto $Y$, et al. Porous-type tracheal prosthesis sealed with collagen sponge. Ann Thorac Surg. 1997;64:965-9.

12. Jones RE, Morgan RF, Marcella KL, et al. Tracheal reconstruction with autogenous jejunal microsurgical transfer. Ann Thorac Surg. 1986;41:636-8.

13. Nakayama M. Experimental reconstruction of the trachea with free jejunal graft. Nippon Kyobu Geka Gakkai Zasshi. 1990;38:1429-35.

14. Khouri RK, Upton J, Shaw WW. Principles of flap prefabrication. Clin Plast Surg. 1992;19:763-71.

15. Papp C, McCraw JB, Arnold PG. Experimental reconstruction of the trachea with autogenous materials. J Thorac Cardiovasc Surg. 1985; 90:13-20.

16. Cavadas PC. Tracheal reconstruction using a free jejunal flap with cartilage skeleton: experimental study. Plast Reconstruct Surg. 1998; 101:937-42.

17. Wheatley MJ, Meltzer TR, Cohen JI. Radial forearm free flap tracheal reconstruction after parastomal tumor resection. Plast Reconstruct Surg. 1998;101:1342-4.

18. Azizzadeh B, Yafai S, Rawnsley JD, et al. Radial forearm free flap pharyngoesophageal reconstruction. Laryngoscope. 2001;111:807-10.

19. Miyazawa T, Yamakido M, Ikeda S, et al. Implantation of ultraflex nitinol stents in malignant tracheobronchial stenoses. Chest. 2000;118:959-65.

20. May A, Hahn EG, Ell C. Self-expanding metal stents for palliation of malignant obstruction in the upper gastrointestinal tract. Comparative assessment of three stent types implemented in 96 implantations. J Clin Gastroenterol. 1996;22:261-6.

21. Rabkin DJ, Lang EV, Brophy DP. Nitinol properties affecting uses in interventional radiology. J Vasc Interv Radiol. 2000;11:343-50.

22. Madden BP, Datta S, Charokopos N. Experience with Ultraflex expandable metallic stents in the management of endobronchial pathology. Ann Thorac Surg. 2002;73:938-44.

23. Rafanan AL, Mehta AC. Stenting of the tracheobronchial tree. Radiol Clin N Am. 2000;38:395-408.

24. Wang MQ, Sze DY, Wang ZP, et al. Delayed complications after esophageal stent placement for treatment of malignant esophageal obstructions and esophagorespiratory fistulas. J Vasc Interv Radiol. 2001;12:465-74. 